adalimumab were more pronounced among patients with higher baseline serum IL-6 levels.

Acknowledgement: Study funding and editorial support (Helen Johns, Adelphi) were provided by Sanofi and Regeneron Pharmaceuticals, Inc. These data have been submitted to the 2019 APLAR-ARA annual meeting.

Disclosure of Interests: Mark C. Genovese Grant/research support from: Sanofi/Genzyme, Genentech/Roche, RPharm, Consultant for: Sanofi/Genzyme, Genentech/Roche, RPharm, Gerd Rüdiger Burmester Consultant for: Roche, Sanofi-Genzyme, Speakers bureau: Roche, Sanofi-Genzyme, Owen Hagino Shareholder of: Sanofi Genzyme, Employee of: Sanofi Genzyme, Hubert van Hoogstraten Shareholder of: Sanofi, Regeneron, Novartis, Grant/research support from: Zambon, Employee of: Sanofi, Erin Mangan Shareholder of: Regeneron, Pfizer, Employee of: Regeneron, Karthinathan Thangavelu Shareholder of: Sanofi, Employee of: Sanofi, Roy Fleischmann Grant/research support from: AbbVie, Amgen, AstraZeneca, BristolMyers Squibb, Celtrion, Genentech, GSK, Janssen, Lilly, Novartis, Pfizer Inc, Sanofi-Aventis, UCB, Consultant for: AbbVie, Amgen, AstraZeneca, Bristol-Myers Squibb, Celtrion, Genentech, GSK, Janssen, Lilly, Novartis, Pfizer Inc, Sanofi-Aventis, UCB, Thomas Mandrup-Poulsen Consultant for: Sanofi Genzyme, Speakers bureau: Sanofi DOI: 10.1136/annrheumdis-2019-eular.3259

\section{SAT0122 COMPARISON OF REAL-WORLD PERSISTENCE OF SUBCUTANEOUSLY ADMINISTERED BIOLOGIC DISEASE-MODIFYING ANTIRHEUMATIC DRUG (DMARD) THERAPIES AMONG PATIENTS WITH RHEUMATOID ARTHRITIS (RA) SWITCHING FROM ANOTHER BIOLOGIC}

Jennie H. Best, Lenore Tominna, Ibrahim Abbass. Genentech, Inc., South San Francisco, United States of America

Background: The EULAR and ACR clinical guidelines recommend switching to a different disease-modifying antirheumatic drug (DMARD) when biologic-treated patients experience treatment failure or toxicity. Lack of efficacy and adverse events are among the most commonly reported reasons for switching biologic therapies. Limited information is available regarding biologic therapy persistence across subcutaneously administered (SC) biologic agents in the real-world setting, as well as for comparative information on biologic persistence for SC biologics among patients with rheumatoid arthritis (RA) who are not naive to biologic treatment.

Objectives: To compare persistence of SC biologic DMARDs (bDMARDs) in patients with RA as subsequent-line therapy following a failure of first line bDMARDs.

Methods: US administrative claims data were used to create a longitudinal cohort of adult patients with RA initiating SC biologic between 1/1/ 2012 and 6/30/2017 (initiation date $=$ index). Patients were required to have failed 1st bDMARD to enter study (but could later switch therapy) and to have 6 and at least 3 months of continuous enrollment pre- and post-index (date of prescription for bDMARD). Those with other autoimmune conditions were excluded from the study. Outcomes were biologic persistence, defined as number of days between initiation date and last supplied day of last fill. Parametric survival models with exponential distribution with robust variance estimator were used to compare outcomes for tocilizumab versus other biologics, adjusting for differences in baseline characteristics, accounting for correlation among different bDMARD episodes.

Results: There were 10,301 patients with 12,704 bDMARD episodes: abatacept $(n=2,988)$, adalimumab $(n=3,599)$, certolizumab $(n=982)$, etanercept $(n=2,760)$, golimumab $(n=745)$, or tocilizumab $(n=1,630)$. Mean age was 51.0-53.3. Mean [SD] Elixhauser comorbidity scores were significantly higher $(p<0.001)$ for tocilizumab (2.8 [2.3]) compared to abatacept $(2.5$ [2.2]), adalimumab (2.5 [2.1]), certolizumab (2.4 [2.0]), etanercept (2.4 [2.0]), or golimumab (2.4 [2.2]). Adjusted median days $(95 \% \mathrm{Cl})$ of persistence were: abatacept 320 (305, 335); adalimumab 280 (268, 293); certolizumab 262 (241, 284); etanercept 289 (274, 304); golimumab 304 $(274,333)$; and tocilizumab $333(311,356)$. Tocilizumab had significantly $(p<0.05)$ higher persistence compared to adalimumab, certolizumab, and etanercept (Figure 1).. Of patients who were observed for 12 months, $45 \%$ of patients initiated tocilizumab bi-weekly and $55 \%$ initiated weekly. Of the 347 patients initiating bi-weekly tocilizumab, $33 \%$ switched to weekly over 12-month follow up; the mean time to switch was 177 days. After 12 months of follow-up, approximately $68 \%$ of patients finished on weekly dosing and $32 \%$ on biweekly dosing.

Conclusion: Among patients with RA who previously used $\geq 1$ other biologic, tocilizumab-treated patients had similar or statistically significantly better biologic persistence compared with other biologics.

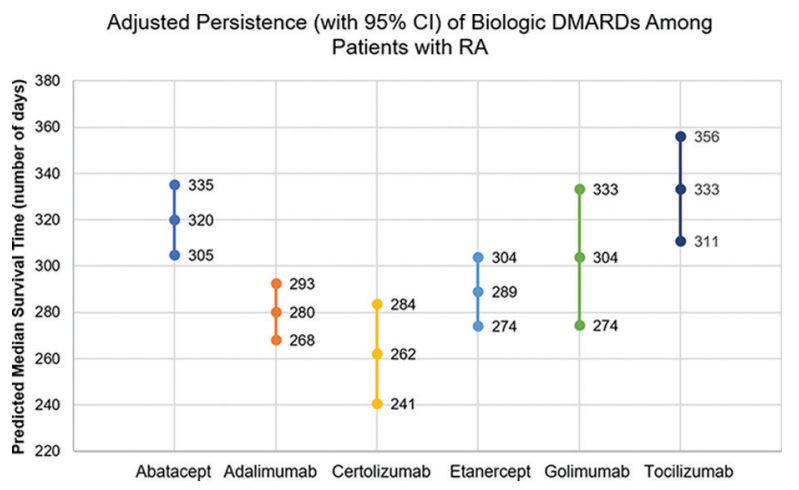

Acknowledgement: This study was funded by Genentech, Inc.

Disclosure of Interests: Jennie H. Best Employee of: Genentech, Inc. Lenore Tominna Employee of: Genentech, Inc., Ibrahim Abbass Employee of: Genentech, Inc.

DOI: 10.1136/annrheumdis-2019-eular.2127

\begin{tabular}{|l|l}
\hline SAT0123 & RISK FOR TUBERCULOSIS DURING TREATMENT WITH \\
BIOLOGICAL THERAPY: IS IT TIME FOR REVIEWING \\
SCREENING PROTOCOL? - RESULTS FROM \\
BRAZILIAN REGISTRY OF BIOLOGICAL THERAPIES IN \\
RHEUMATIC DISEASES (BIOBADABRASIL)
\end{tabular}

Ana Medeiros, M Bredemeier, V Valim, M Pinheiro, C Macieira, A Duarte, B Stadler, R Ranza, M Bertolo, J Miranda, C Brenol, G Castro, V Fernandes, D Titton, F Sauma, I Pereira, R Botelho, H Carvalho, A Hayata, P Louzada, A Ranzolin, S Studart, G Castelar, A Kakehasi, W Bianchi, R Acayaba, I Silveira, H Resende, J Amaral, L Rocha, M Gazzeta, L Carvalho, S Schowalski, D Feldman, I Laurindo, J Provenza, Brazilian Society of Rheumatology. BiobadaBrasil, São Paulo, Brazil

Background: As Brazil is accountable for $33 \%$ of the tuberculosis (TB) burden in Americas, with an annual incidence of 33.5/100,000 in 2017[1], the occurrence of tuberculosis infection in patients with rheumatic diseases in use of biological therapy is a recurrent concern.

Objectives: To assess incident TB among patients with rheumatic disease in use of biological therapy in a country with high incidence of TB.

Methods: BiobadaBrasil is a multicentric prospective cohort study involving patients with rheumatic diseases who started the first biologic or a synthetic disease modifying anti-rheumatic drug (DMARD)[6]. This analysis includes patients with rheumatoid arthritis (RA), psoriatic arthritis (PSA), and ankylosing spondylitis (AS) recruited from Jan 2009 to Aug 2018 and followed-up for one or multiple courses of treatment until censoring or incident TB. The primary outcome was the incidence of tuberculosis (in any organ site). The history of exposure to tuberculosis, chest $\mathrm{Rx}$, screening and treatment for latent tuberculosis infection (LBTI), and use of prophylactic isoniazid were evaluated before each course of treatment. Multivariate Cox proportional hazards models (with DMARDs included as time-varying covariates) were used to estimate hazard ratios (HR) and $95 \%$ confidence intervals $(\mathrm{Cl})$; analyses were performed with the Survival package of $R$.

Results: Sample: 2858 patients (female $=70,2 \%$; $R A=72 \%, A S=20 \%$, and PsA $=8 \%)$. A total of $31(1.1 \%)$ patients developed tuberculosis during treatment. One patient on abatacept and one on tocilizumab developed TB while all the others were on TNF-inhibitors(29). The median (interquartile range) exposure to the current DAMRD course was 11.1 (5.9-20) months. TB patients did not significantly differ from others regarding disease duration, sex or age. Almost half $(n=14,45 \%)$ of TB patients did not present evidence of previous exposure or any screening positive test for TB; 9 (29\%) TB patients had received adequate LTBI treatment (isoniazid) recently or in the past. Furthermore, $8(26 \%)$ had incomplete/ inconsistent screening. The overall incidence of TB was 1.9/1000 patients/year, and was not significantly different among the diseases (1.63 for RA, 2.06, for AS, and 3.79/1000/year for PsA). In univariate analysis, exposure to anti-TNF monoclonal antibodies (HR $3.1,95 \% \mathrm{Cl} 1.18-8.15$, $\mathrm{P}=0.02$ ) and presence of any marker of previous contact with TB (positive history of TB, known contact with TB, positive TST, or abnormal chest X-ray: HR $4.2(2.1-8.5, p<0,001)$ were positively associated with the risk of TB. Including simultaneously these variables in the Cox model, they were independent risk factor for TB $(p<0.05)$.

Conclusion: Monoclonal anti-TNF antibodies and previous exposure/diagnosis of TB are independent risk factors for developing TB in Brazil. TB 
cases occurred both early and lately during treatment courses, suggesting LTBI screening failures, treatment non-adherence or re-exposure. Current application and content of the protocol for screening and treatment of LTBI needs to be reviewed.

\section{REFERENCES}

[1] WHO TB report 2018.https://www.who.int/tb/publications/global_report/en/

[2] Rev Bras Reumatol Engl Ed. 2017;57 Suppl 2:477-483. doi: 10.1016/j. rbre.2017.05.005.

Acknowledgement: monitor $\mathrm{P}$ Cabral, MDs $\mathrm{N}$ Sacilotto, $\mathrm{H}$ Pereira, $\mathrm{F}$ Sztajnbok.

Disclosure of Interests: Ana Medeiros: None declared, M Bredemeier: None declared, V Valim: None declared, M Pinheiro Consultant for: Janssen, Pfizer, Speakers bureau: Abbot,Janssen,Novartis, C Macieira: None declared, A Duarte: None declared, B Stadler: None declared, R Ranza: None declared, M Bertolo: None declared, J Miranda Speakers bureau: Pfizer, C Brenol Speakers bureau: Pfizer, Roche, Janssen, Bristol., G Castro: None declared, V Fernandes Speakers bureau: Janssen, D Titton: None declared, F Sauma: None declared, I Pereira: None declared, R Botelho: None declared, H Carvalho: None declared, A Hayata: None declared, P Louzada: None declared, A Ranzolin: None declared, S Studart: None declared, G Castelar: None declared, A Kakehasi Grant/ research support from: Abbvie, UCB, JANSSEN, ROCHE, NOVARTIS, PFIZER, Consultant for: Abbvie, UCB, JANSSEN, ROCHE, NOVARTIS, PFIZER, Paid instructor for: JANSSEN, Speakers bureau: Abbvie, UCB, JANSSEN, ROCHE, NOVARTIS, PFIZER, W Bianchi: None declared, R Acayaba: None declared, I Silveira: None declared, H Resende: None declared, J Amaral: None declared, L Rocha: None declared, M Gazzeta: None declared, L Carvalho: None declared, S Schowalski: None declared, D Feldman: None declared, I Laurindo Consultant for: Abbvie, UCB, GSK, JANSSEN, LILLY, NOVARTIS, PFIZER, Paid instructor for: Abbvie, JANSSEN, Speakers bureau: Abbvie, UCB, GSK, JANSSEN, LILLY, NOVARTIS, PFIZER, ROCHE, J Provenza: None declared

DOI: 10.1136/annrheumdis-2019-eular.4624

\section{SAT0124 MOLECULAR CHARACTERIZATION OF THE SERUM PROFILE ASSOCIATED TO THE INCREASED CARDIOVASCULAR RISK IN RHEUMATOID ARTHRITIS PATIENTS. EFFECTS OF BIOLOGICAL DRUGS.}

Carlos Perez-Sanchez ${ }^{1}$, Nuria Barbarroja Puerto ${ }^{1}$, Pérez Sánchez Laura ${ }^{1}$, Patricia Ruiz-Limon ${ }^{2}$, Sara Remuzgo Martinez ${ }^{3}$, Alejandro Ibañez-Costa ${ }^{1}$, Maria Luque-Tevar ${ }^{1}$, Alejandra M. Patiño-Trives ${ }^{1}$, Iván Arias de la Rosa ${ }^{1}$, Maria del Carmen Abalos-Aguilera ${ }^{1}$, Rafaela Ortega Castro ${ }^{1}$, Alejandro

Escudero Contreras ${ }^{1}$, Raquel López-Mejías ${ }^{3}$, M Ángeles Aguirre-Zamorano ${ }^{1}$, Eduardo Collantes Estevez ${ }^{1}$, Miguel A. González-Gay ${ }^{3}$, Chary Lopez-Pedrera'1. ${ }^{1}$ IMIBIC/Reina Sofia Hospital/University of Cordoba, Cordoba, Spain, ${ }^{2}$ IBIMA Hospital Virgen de la Victoria, Malaga, Spain, ${ }^{3}$ IDIVAL/Marques de Valdecilla Hospital/University of Cantabria, Santander, Spain

\section{Background:}

Objectives: 1- To characterize the serum molecular profile associated to the increased cardiovascular (CV) risk in Rheumatoid Arthritis (RA) patients. 2- To evaluate the in vivo and in vitro effects of biological drugs on the reestablishment of this altered molecular profile.

Methods: Serum samples of 280 RA patients and 100 healthy donors (HD) were studied. miRNomes were identified using next-generation sequencing miRNA assay (HTG EdgeSeq technology). The inflammatory profile, Netosis-derived products, and circulating biomolecules related to oxidative stress were quantified using commercial kits. The Cardiovascular Risk SCORE for RA patients was calculated following EULAR recommendations. Carotid intima-media thickness (CIMT) was evaluated as early atherosclerosis marker. The in vivo effects of biologic drugs such as Infliximab (IFX), Tocilizumab (TCZ) and Rituximab (RTX) were evaluated before and after 6 months of therapy in 45,20 and 25 RA patients, respectively. Serum from RA patients with high and low CV risk scores either before and after IFX, TCZ and RTX therapies-, were further added to HUVECs, monocytes, and neutrophils purified from HD, and activity profiles were evaluated.

Results: The miRNA whole transcriptome assay identified 104 circulating miRNAs altered in RA patients. Functional classification (IPA) established their involvement in inflammatory response, as well as in immunological and hematological diseases. Circulating biomolecules related to inflammation -interleukins, chemokines, adhesion molecules-, Netosis -cell-free nucleosomes, elastase and DNA- and oxidative stress -lipoperoxides, nitrated proteins, and total antioxidant capacity- were also found coordinately altered in the serum of RA patients. Multivariate analyses showed that levels of a number of those altered biomolecules and circulating microRNAs were predictors of a high Cardiovascular Risk SCORE and the presence of a pathologic CIMT in RA patients.

The in vivo treatments with IFX, TCZ and RTX for six months reduced disease activity and induced the re-establishment of normal levels in those altered biomolecules in RA patients. Mechanistic in vitro studies showed increased pro-inflammatory profiles of leukocytes subsets and HUVECSs after treatment with serum from high CV risk score-RA patients. These profiles were reversed by incubation with serum from those patients after biologic drugs treatment.

Conclusion: 1. Specific mediators of inflammation, oxidative damage and Netosis, along with the microRNAs modulating their expression, coordinately contribute to the higher CV risk score present in RA patients. 2. Biologic drugs such as IFX, TCZ and RTX, restore the normal levels of these altered biomolecules, reducing the $\mathrm{CV}$ risk in RA patients.

Acknowledgement: Funded by PI-0285-2017, ISCIII, PI18/00837 and RIER RD16/0012/0015 co-funded with FEDER

Disclosure of Interests: Carlos Perez-Sanchez: None declared, Nuria Barbarroja Puerto: None declared, Pérez Sánchez Laura: None declared, Patricia Ruiz-Limon: None declared, Sara Remuzgo Martinez: None declared, Alejandro Ibañez-Costa: None declared, Maria Luque-Tevar: None declared, Alejandra M. Patiño-Trives: None declared, Iván Arias de la Rosa: None declared, Maria del Carmen Abalos-Aguilera: None declared, Rafaela Ortega Castro: None declared, Alejandro Escudero Contreras: None declared, Raquel López-Mejías: None declared, M Ángeles Aguirre-Zamorano: None declared, Eduardo Collantes Estevez: None declared, Miguel A González-Gay Grant/research support from: Prof. MA Gonzalez-Gay received grants/research supports from Abbvie, MSD, Jansen and Roche., Speakers bureau: Consultation fees/participation in company sponsored speaker's bureau from Pfizer, Lilly, Sobi, Celgene, Novartis, Roche and Sanofi., Chary Lopez-Pedrera: None declared DOI: 10.1136/annrheumdis-2019-eular.7338

\section{SAT0125 LONG-TERM SAFETY WITH SARILUMAB PLUS CONVENTIONAL SYNTHETIC DISEASE-MODIFYING ANTIRHEUMATIC DRUGS AND SARILUMAB MONOTHERAPY IN RHEUMATOID ARTHRITIS: AN INTEGRATED ANALYSIS WITH 9,000 PATIENT-YEARS OF FOLLOW-UP}

Roy Fleischmann ${ }^{1}$, Yong Lin² ${ }^{2}$ Gregory St John ${ }^{3}$, Désirée van der Heijde ${ }^{4}$, Chunfu Qiu ${ }^{2}$, Juan Jose Gomez-Reino ${ }^{5}$, Jose Antonio Maldonado-Cocco ${ }^{6}$ Marina Stanislav ${ }^{7}$, Bruno Seriolo ${ }^{8}$, Gerd Rüdiger Burmester ${ }^{9}{ }^{1}$ Metroplex Clinical Research Center, Dallas, TX, United States of America; ${ }^{2}$ Sanofi Genzyme, Bridgewater, NJ, United States of America; ${ }^{3}$ Regeneron Pharmaceuticals Inc, Tarrytown, NY, United States of America; ${ }^{4}$ Leiden University Medical Center, Leiden, Netherlands; ${ }^{5}$ Complejo Hospitalario Universitario de Santiago, Santiago, Spain; ${ }^{6}$ Buenos Aires University, Buenos Aires, Argentina; ${ }^{7}$ Research Rheumatology Institute n. a. V.A. Nassonova, Moscow, Russian Federation; ${ }^{8}$ University of Genova, Genova, Italy, ${ }^{9}$ Charité - University Medicine Berlin, Berlin, Germany

Background: Sarilumab, a human IL-6R blocker approved for the treatment of RA, has shown efficacy as monotherapy and in combination with csDMARDs in Phase 3 trials.

Objectives: We assessed long-term safety from the sarilumab clinical development program in adult patients with RA who received subcutaneous (SC) sarilumab in eight clinical trials and their open-label extensions: MOBILITY (NCT01061736), TARGET (NCT01709578), ASCERTAIN (NCT01768572), EASY (NCT02057250), COMPARE (NCT01764997), ACT11575 (NCT01217814), MONARCH (NCT02332590), ONE (NCT02121210), and the open-label extension EXTEND (NCT01146652). Methods: Data (cut-off Jan 15, 2018) were pooled from patients on sarilumab+csDMARD $(\mathrm{N}=2887)$ or sarilumab monotherapy $(\mathrm{N}=471)$. Patients had received sarilumab $200 \mathrm{mg}$ or $150 \mathrm{mg} \mathrm{q} 2 \mathrm{w} \mathrm{SC}$, except for 151 patients from MOBILITY Part A who received $100 \mathrm{mg}$ qw, $150 \mathrm{mg}$ qw, or $100 \mathrm{mg} \mathrm{q} 2 \mathrm{w}$. Treatment-emergent (TE) adverse events (AEs), AEs of special interest (AESIs), and discontinuations were assessed. 